\title{
Serum fetuin-A levels are associated with carotid intima-media thickness in patients with normotensive chronic obstructive pulmonary disease
}

\author{
Seref Alpsoy, Aydin Akyuz, Levent Cem Mutlu, Mustafa Oran, \\ Dursun Cayan Akkoyun, Birol Topcu, Hasan Degirmenci, Savas Guze \\ Namık Kemal University, Faculty of Medicine, Tekirdağ, Turkey
}

\begin{abstract}
Background: There are contradictory reports about the relationship between fetuin- $A$ and atherosclerotic process. Coronary artery disease is the most important cause of mortality in patients with chronic obstructive pulmonary disease (COPD). We aimed to investigate the association of serum fetuin-A level with mean carotid intima-media thickness (cIMT) and ankle-brachial index (ABI) in COPD.

Methods: We evaluated the association of serum fetuin-A level, mean cIMT and ABI in normotensive subjects with COPD $(n=65)$ and with non-COPD $(n=50)$.

Results: Fetuin-A level was significantly lower $(63.5 \pm 19.8 \mathrm{ng} / \mathrm{mL}, 72.9 \pm 16.2 \mathrm{ng} / \mathrm{mL}$, $p=0.035)$ and $C$-reactive protein level higher (4 [1-10] vs. $3[1-12] \mathrm{mg} / \mathrm{dL}, p=0.034$ ) in COPD patients than the control group. Compared to controls, fetuin-A level was significantly lower (63.5 $\pm 19.8 \mathrm{ng} / \mathrm{mL}, 72.9 \pm 16.2 \mathrm{ng} / \mathrm{mL}, p=0.035)$ and mean cIMT higher $(0.69$ [0.50-0.98] vs. 0.62 [0.44-0.98] $\mathrm{mm}, p=0.034$, respectively) in the COPD group. There was a significant negative correlation between mean cIMT and fetuin-A levels $(r=-0.320$, $p=0.032)$. Age $(\beta \pm S E: 0.002 \pm 0.001, p=0.008)$ and fetuin- $A(\beta \pm S E:-0.002 \pm 0.001$, $p=0.035)$ were decisive for the mean cIMT.

Conclusions: There are increased cIMT values, decreased fetuin-A levels, but unchanged $A B I$ values in patients with normotensive COPD. Age and fetuin-A were predictors for cIMT, while fetuin-A was negatively correlated with cIMT. (Cardiol J 2014; 21, 2: 191-197)
\end{abstract}

Key words: carotid intima-media thickness, chronic obstructive pulmonary disease, fetuin-A

\section{Introduction}

Chronic obstructive pulmonary disease (COPD) is an important cause of morbidity and mortality all over the world, and occurs as a result of an abnormal, irreversible and progressive inflammatory response of airways to smoking and noxious particles or gases [1]. Atherosclerosis and coronary artery disease (CAD) are some of the co-morbidities of COPD, which is considered part of a chronic systemic inflammatory syndrome. COPD is a strong risk factor for cardiovascular (CV) events and doubles the risk of CV morbidity and mortality [2,3]. There is a relationship between atherosclerosis

Address for correspondence: Aydın Akyuz, MD, Namık Kemal University, Faculty of Medicine, Tekirdağ, Turkey, e-mail: ayakyuzq5@gmail.com

Received: 07.03.2013 Accepted: 12.05.2013 
and inflammation which plays an important role in all stages of atherosclerosis. Many studies have shown that similar inflammatory markers, such as tumor necrosis factor (TNF) and interleukin 6 (IL-6), increase in COPD and atherosclerosis [4-8].

Fetuin-A is a blood protein whose molecular weight is $60 \mathrm{kDa}$ and which is secreted from the liver. A potent inhibitor of systemic calcification, fetuin-A has a role in metabolic pathways such as calcium homeostasis, insulin sensitivity, promoting phagocytosis of apoptotic cells and bone development [9-11]. The association of lower fetuin-A, which is a novel biomarker, with COPD [12], late macrovascular complications in diabetic patients and subclinical atherosclerosis has been recently investigated [13-15]. The carotid intima-media thickness (cIMT) measurement, which is a non-invasive tool to track changes in arterial walls, is used to detect the presence of early subclinical atherosclerosis [16]. We sought to determine whether there was any relationship between cIMT and fetuin-A levels in patients with COPD; such a relationship has not been well documented in the literature.

\section{Methods}

\section{Subjects}

This study was carried out in our cardiology and chest outpatient clinics between September 2010 and October 2012. One hundred and thirty subjects were initially selected for the study and they were divided into two groups of COPD and control. The study group was composed of 80 patients with COPD, between 35 and 65 years of age. Eight people with CAD and 7 people with heart failure were excluded from the study. As a result, 65 patients made up the sample. There were 36 subjects with dyslipidemia and 41 subjects were smokers in the patient group. There were no hypertensive and diabetic subjects in the COPD group. The control group consisted of 50 non-COPD subjects with age, sex and CV risk factors matched to the COPD group. There were 26 subjects with dyslipidemia in the control group. The control group did not include diabetic or hypertensive individuals or smokers. Subjects with dyslipidemia in both groups were taking statin medication. Patients with heart failure, diabetes mellitus, chronic liver disease, kidney failure, heart valve disease, peripheral artery disease, and CAD were excluded from the study. For the study, ethical approval was obtained from the local ethics committee and written informed consent was obtained from each patient in order to participate in the study.

\section{Study protocols}

Baseline characteristics such as age, gender, height, weight, body mass index, smoking, hypertension, diabetes and hyperlipidemia were recorded for the study and control groups. Patients were asked to fast overnight at least $12 \mathrm{~h}$; afterwards, antecubital venous blood samples were collected for the measurement of fasting glucose, total cholesterol (TC), triglyceride (TG), high density lipoprotein cholesterol (HDL-C), low density lipoprotein cholesterol (LDL-C), C-reactive protein (CRP) and fetuin-A. For fetuin-A measurements blood was collected in separate tubes and centrifuged, plasma was separated and stored at $-80^{\circ} \mathrm{C}$.

\section{Biochemical analyses}

Following an overnight fast, blood samples were collected for biochemical analysis at same time intervals (8:00 to 11:00). A standard enzymatic method using an auto-analyzer (AU680 auto-analyzer [Beckman Coulter, Brea, CA]) was used for the measurement of fasting glucose, TC, HDL-C, LDL-C and TG levels. A standard nephelometry method was used for the measurement of CRP levels (Cobas 311, Roche Diagnostics, Mannheim, Germany) with a sensitivity of $0.1 \mathrm{mg} / \mathrm{L}$. Blood samples taken for fetuin-A were centrifuged and the separated serum samples stored at $-80^{\circ} \mathrm{C}$ until they were analyzed.

\section{Fetuin-A assay}

Serum concentrations of fetuin-A were measured by using a human fetuin-A ELISA kit (BioVendor Laboratory Medicine, Inc., Brno, Czech Republic) in an ELISA plate reader (ELX 800 Multi-Plate Reader, Bio-tek Instruments, Inc., Winooski, VT, USA). Performance data were provided by the manufacturer. The minimum detectable concentration for fetuin-A was $5 \mathrm{ng} / \mathrm{mL}$. The measured intra-assay and inter-assay variability coefficients were $3.9 \%$ and $2.6 \%$, respectively. Reference range in healthy subjects has not been defined by the manufacturer yet. A study reveals its normal range as $15-90 \mathrm{ng} / \mathrm{mL}$, however, there is poor agreement between plasma fetuin-A measurements made using different ELISA methods [17].

\section{Pulmonary function tests}

Pulmonary function tests included forced expiratory volume in $1 \mathrm{~s}\left(\mathrm{FEV}_{1}\right)$ and forced vital capacity (FVC); $\mathrm{FEV}_{1} / \mathrm{FVC}$ were measured with 
Jaeger MasterScope spirometer (VIASYS Healthcare, Germany). Tests were repeated at least 3 times in the sitting position and the best test was accepted as the final result to evaluate the percentage of values predicted by the spirometer's software program. COPD was diagnosed according to the criteria of the Global Initiative for Chronic Obstructive Lung Disease (GOLD). The protocol for lung function measurements conformed to the American Thoracic Society recommendations [18]. According to their pulmonary function test, 17 patients were assigned stage I, 38 patients - stage II, 6 patients - stage III and 4 patients - stage IV. After examination in the chest outpatient clinic, patients were invited to cardiology outpatient clinic for the measurement of cIMT and ankle-brachial index (ABI).

\section{Ultrasonographic measurement of the cIMT and ABI parameters}

Ultrasonographic and echocardiographic measurements were performed by a cardiologist who was unaware of the case-control status of the subjects. Three different measurements for cIMT were performed, and the average was taken.

\section{Carotid intima-media thickness measurements}

All participants were at rest for $10 \mathrm{~min}$ before the examination, and placed in the supine position with their head tilted backwards. The right and left common carotid arteries were measured with real time scanner and with a synchronized ECG (Esaote MyLab 50, Genoa, Italy) after visualization using a $7.5 \mathrm{MHz}$ linear array transducer using the semi-automated-guided technique radiofrequency-Quality Intima Media Thickness (QIMT; Esaote, Maastricht, Holland). A 1-cm segment was focused in the region of the first $2 \mathrm{~cm}$ below the common carotid artery bulb, and posterior wall IMT was measured. cIMT was defined as the average of the right and left cIMT.

\section{Ankle brachial pressure index measurements}

After the patients were at rest for 5-10 $\mathrm{min}$ in the supine position with the patient's arms and legs at the same level as the heart, a pneumatic cuff was placed above the antecubital fossa, and an $8 \mathrm{mHz}$ continuous wave hand-held Doppler probe (Sonotrax $-8 \mathrm{MHz}$ Sonotrax Ultrasonic Vascular Pocket Doppler, stork RADIO LLC, Boca Raton, FL, USA) was placed on the brachial artery. After the pneumatic cuff was inflated to at least $20 \mathrm{~mm} \mathrm{Hg}$ above suprasystolic pressure, cuff pressure was gradually reduced. The first sound heard was recorded as brachial systolic blood pressure (BP). This was repeated for the other arm and the highest one was used for the calculation of ABI. To measure ankle systolic BP, the BP cuff was placed $5 \mathrm{~cm}$ above the medial malleolus on the each ankle. Then, both lower extremities' systolic BP were measured in the same way for the dorsalis pedis and tibialis posterior artery. The $\mathrm{ABI}$ is calculated by dividing the higher systolic $\mathrm{BP}$ at the ankle by the higher systolic BPs in the arm. From the calculated results, the lowest value was defined as the ABI. An ABI value less than 0.9 was considered to represent peripheral vascular disease $[19,20]$.

The cIMT and ABI measurements of 20 patients with COPD were re-examined after 10 days to determine the coefficients of variation between visits. These were $3.5 \%$ and $3.1 \%$ for cIMT and $\mathrm{ABI}$, respectively.

\section{Statistical analysis}

Data were analyzed using the Predictive Analysis Software (PASW) Statistics 18 (SPSS Inc., Chicago, IL, USA). A Kolmogorov-Smirnov test was used to check continuous variable distributions. Descriptive statistics were expressed as mean \pm standard deviation for the parametric data, or median (interquartile range) for the non-parametric data. For the comparison of the two groups, the independent t-test was used for normally distributed variables, whereas the Mann-Whitney U test was used for non-normally distributed variables, while the $\chi^{2}$ test was used for categorical variables. Spearman rank correlation analysis was employed to estimate the relationship between test parameters. A p value $<0.05$ was considered statistically significant. After entering univariate predictors with $\mathrm{p}<0.2$, multivariate linear regression analysis was performed to detect the independent variables affecting cIMT parameters.

\section{Results}

\section{Anthropometric, clinical features and biochemical variables}

A total of 65 patients with COPD (39 male, $60 \%$ ), and 50 non-COPD control subjects (29 male, $58 \%$ ) were evaluated. The mean duration of the disease and the mean duration of smoking of the study group was $11 \pm 4.2$ and $28 \pm 4.7$ years, respectively. The mean age, male percentage and body mass index of the COPD group and the control group were similar $(61.7 \pm 10.6$ vs. $60.2 \pm 12.9$ years, 
Table 1. Anthropometric, clinical features, and biochemical parameters of the chronic obstructive pulmonary disease (COPD) and control groups.

\begin{tabular}{|c|c|c|c|}
\hline & COPD group $(n=65)$ & Controls group $(n=50)$ & $\mathbf{P}^{*}$ \\
\hline Age [years] & $61.7 \pm 10.6$ & $60.2 \pm 12.9$ & 0.248 \\
\hline Gender, male & $39(60 \%)$ & $29(58 \%)$ & 0.141 \\
\hline Body mass index $\left[\mathrm{kg} / \mathrm{m}^{2}\right]$ & $26 \pm 3.5$ & $25.8 \pm 2.6$ & 0.865 \\
\hline Current smoker & $41(63 \%)$ & $0(0 \%)$ & $<0.001$ \\
\hline Duration of smoking [years] & $28 \pm 4.7$ & $0(0 \%)$ & $<0.001$ \\
\hline Duration of disease [years] & $11 \pm 4.2$ & $0(0 \%)$ & $<0.001$ \\
\hline Dyslipidemia & $36(55.3 \%)$ & $26(52 \%)$ & 0.564 \\
\hline $\mathrm{FEV}_{1}[\%]$ & $61.1 \pm 17.6$ & $100.2 \pm 12.1$ & $<0.001$ \\
\hline FVC [\%] & $84.8 \pm 18.6$ & $96.4 \pm 9.5$ & 0.002 \\
\hline $\mathrm{FEV}_{1} / \mathrm{FVC}$ & $55.7(35-81)$ & $86.4(68-95)$ & $<0.001$ \\
\hline Systolic blood pressure [mm Hg] & $126 \pm 7$ & $128 \pm 6$ & 0.563 \\
\hline Diastolic blood pressure [mm Hg] & $81 \pm 2.7$ & $79 \pm 2.9$ & 0.441 \\
\hline Total cholesterol [mg/dL] & $200(115-318)$ & $191(121-260)$ & 0.726 \\
\hline Triglycerides [mg/dL] & $124(43-368)$ & $161(52-388)$ & 0.394 \\
\hline $\mathrm{HDL}-\mathrm{C}[\mathrm{mg} / \mathrm{dL}]$ & $44(18-69)$ & $42(30-181)$ & 0.806 \\
\hline LDL-C [mg/dL] & $137 \pm 39$ & $130 \pm 37$ & 0.472 \\
\hline Uric acid [mg/dL] & $6.5 \pm 3.3$ & $6.6 \pm 3.8$ & 0.968 \\
\hline Fasting glucose [mg/dL] & $87(53-113)$ & $73(57-113)$ & 0.314 \\
\hline C-reactive protein [mg/dL] & $4(1-10)$ & $3(1-12)$ & 0.034 \\
\hline Fetuin-A [ng/mL] & $63.5 \pm 19.8$ & $72.9 \pm 16.2$ & 0.035 \\
\hline
\end{tabular}

Data are presented as mean $\pm \mathrm{SD}$ and median (interquartile range); * $\chi^{2}$ test, Mann-Whitney $U$ test and Student's $t$ test; FEV ${ }_{1}-$ forced expiratory volume in $1 \mathrm{~s}$; FVC — forced vital capacity; HDL — high density lipoprotein cholesterol; LDL — low density lipoprotein cholesterol

$\mathrm{p}=0.248 ; \mathrm{n}=39[60 \%]$ vs. $\mathrm{n}=29[58 \%]$, $\mathrm{p}=0.141)$ and $\left(26 \pm 3.5\right.$ vs. $25.8 \pm 2.6 \mathrm{~kg} / \mathrm{m}^{2}$, $\mathrm{p}=0.865)$, respectively. Dyslipidemia rates did not differ between the study and control groups $(\mathrm{n}=36$ [55.3\%], $\mathrm{n}=26$ [52\%], $\mathrm{p}=0.564)$. Additionally, there was no difference between the two groups in terms of systolic BP, TC, TG, HDL-C, LDL-C, fasting glucose, uric acid (all values of $\mathrm{p}>0.05$ ). The COPD group had higher CRP levels (4 [1-10] vs. 3 [1-12] mg/L, p = 0.034) and lower fetuin-A levels $(63.5 \pm 19.8$ vs. $72.9 \pm 16.2 \mathrm{ng} / \mathrm{mL}$, $\mathrm{p}=0.035)$ compared to the control group (Table 1 ).

\section{Spirometer values}

The COPD group had a lower $\mathrm{FEV}_{1}(\%)$ $(61.1 \pm 17.6$ vs. $100.2 \pm 12.1, \mathrm{p}<0.001), \mathrm{FVC}$ $(84.8 \pm 18.6$ vs. $96.4 \pm 9.5 \%, \mathrm{p}=0.002), \mathrm{FEV}_{1} /$ /FVC (55.7 [35-81] vs. 86.4 [68-95], p < 0.001) compared to the control group (Table 1).

\section{Carotid IMT and ABI}

Compared with the control group, the COPD group had higher cIMT $(0.69$ [0.50-0.98] vs. 0.62 [0.44-0.98] mm, p = 0.034, respectively). ABI values were not statistically different between the two groups (1.08 [1-1.31] vs. 1.11 [1.0-1.47], $\mathrm{p}=0.278$; Table 2 ). There was a negative correlation between fetuin-A and cIMT values in the COPD group, but not in the control group ( $\mathrm{r}=-0.320$, $\mathrm{p}=0.032$ and $\mathrm{r}=-0.116, \mathrm{p}=0.423$ ) (Figs. 1, 2). No correlation was seen between fetuin-A and ABI values $(r=-0.06, p=0.691)$.

In univariate regression analysis, age, $\mathrm{FEV}_{1}$, systolic BP, LDL-C, fasting glucose, CRP and fetuin-A levels were found as parameters with $\mathrm{p}<0.2$. After these variables, in multivariate regression analysis, the predictors of increased cIMT were age $(\beta \pm$ SE: $0.002 \pm 0.001, \mathrm{p}=0.008)$ and fetuin-A levels $(\beta \pm \mathrm{SE}:-0.002 \pm 0.001$, $\mathrm{p}=0.035)$ (Table 3$)$.

\section{Discussion}

No study so far has investigated the relationship between fetuin-A, cIMT and ABI in patients with COPD. We particularly studied subjects with no manifest atherosclerosis to determine the association between COPD and subclinical atherosclerosis concerning serum fetuin-A levels. The present study revealed the following findings: 
Table 2. The parameters of carotid intima-media thickness and ankle-brachial index values of the chronic obstructive pulmonary disease (COPD) and control groups.

\begin{tabular}{lccc}
\hline & COPD group $(\mathbf{n}=65)$ & Control group $(\mathbf{n}=50)$ & P* \\
\hline CCA-IMT [mm] & $0.69(0.50-0.98)$ & $0.62(0.44-0.98)$ & 0.034 \\
Ankle-brachial index & $1.08(1-1.31)$ & $1.11(1.0-1.47)$ & 0.278 \\
Left ventricular ejection fraction [\%] & $66(50-69)$ & $68(58-74)$ & 0.856 \\
\hline
\end{tabular}

*Mann-Whitney U test; CCA-IMT — common carotid artery intima-media thickness

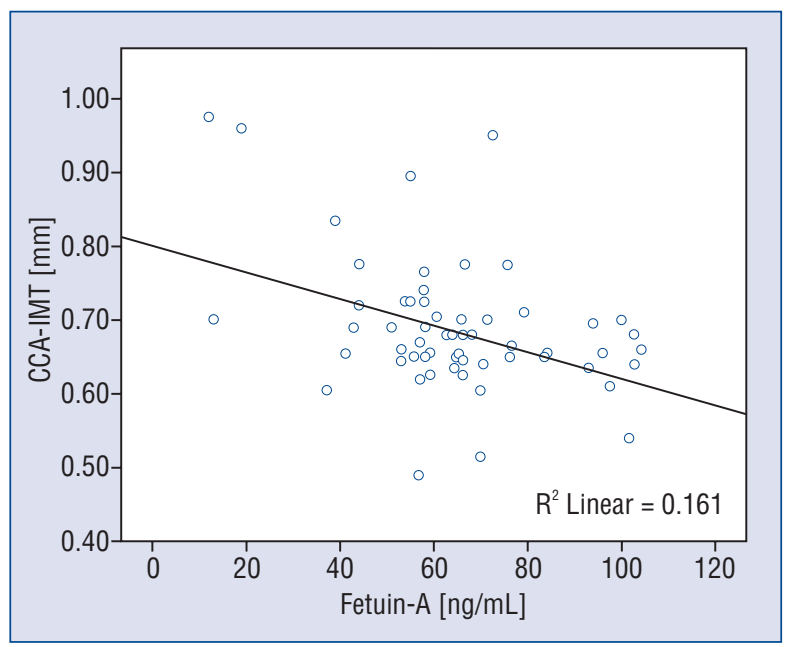

Figure 1. A negative linear correlation between serum fetuin-A levels and increasing mean common carotid artery intima-media thickness (CCA-IMT) is shown on scatter diagram in the chronic obstructive pulmonary disease group (Spearman rank correlation coefficient $r=-0.320, p=0.032$ ).

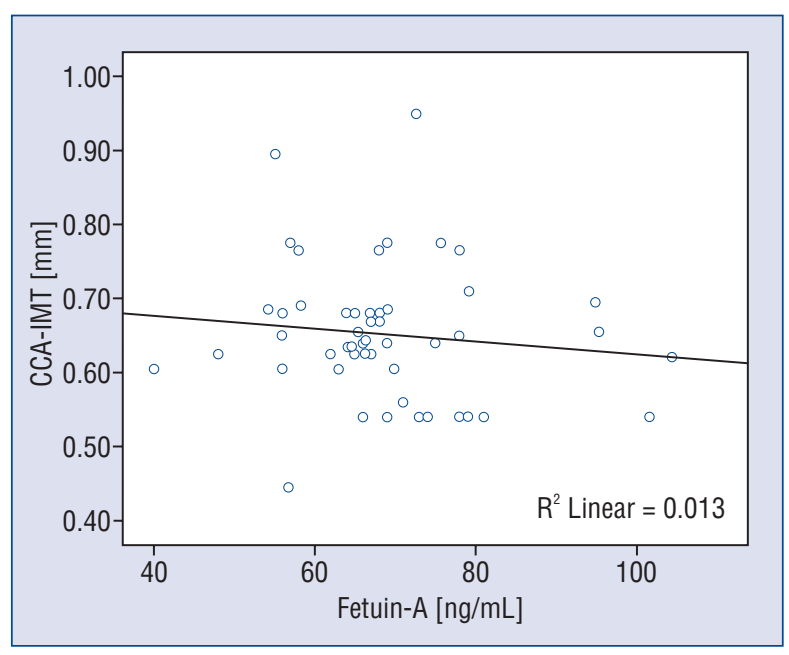

Figure 2. No correlation was shown between mean common carotid artery intima-media thickness (CCA-IMT) and serum fetuin-A levels in the control group (Spearman rank correlation coefficient $r=-0.116$; $p=0.423$ ).
Table 3. Univariate and multivariate analysis for predictors of carotid intima-media thickness.

\begin{tabular}{|c|c|c|}
\hline & $\begin{array}{c}\text { Unstandardized } \\
\beta \pm \mathrm{SE}\end{array}$ & $\mathbf{P}$ \\
\hline \multicolumn{3}{|l|}{ Univariate analysis } \\
\hline Age [years] ${ }^{*}$ & $0.002 \pm 0.001$ & 0.039 \\
\hline Body mass index $\left[\mathrm{kg} / \mathrm{m}^{2}\right]$ & $-0.02 \pm 0.003$ & 0.453 \\
\hline $\mathrm{FEV}_{1}[\%]^{*}$ & $0.001 \pm 0.001$ & 0.150 \\
\hline FVC [\%] & $0.001 \pm 0.001$ & 0.385 \\
\hline $\mathrm{FEV}_{1} / \mathrm{FVC}$ & $0.001 \pm 0.001$ & 0.256 \\
\hline Systolic BP [mm Hg] ${ }^{*}$ & $0.001 \pm 0.001$ & 0.197 \\
\hline Diastolic BP [mm Hg] & $0.001 \pm 0.001$ & 0.432 \\
\hline Total cholesterol [mg/dL] & $0.001 \pm 0.001$ & 0.704 \\
\hline Triglycerides [mg/dL] & $0.001 \pm 0.001$ & 0.923 \\
\hline HDL-C [mg/dL] & $0.001 \pm 0.001$ & 0.878 \\
\hline LDL-C [mg/dL]* & $0.001 \pm 0.002$ & 0.184 \\
\hline Uric acid [mg/dL] & $0.001 \pm 0.002$ & 0.587 \\
\hline Fasting glucose $[\mathrm{mg} / \mathrm{dL}]^{*}$ & $0.001 \pm 0.001$ & 0.182 \\
\hline C-reactive protein $[\mathrm{mg} / \mathrm{dL}]^{*}$ & $-0.002 \pm 0.001$ & 0.129 \\
\hline Fetuin-A [ng/mL] ${ }^{*}$ & $-0.001 \pm 0.001$ & 0.032 \\
\hline \multicolumn{3}{|l|}{ Multivariate analysis } \\
\hline Age [years] & $0.002 \pm 0.001$ & 0.008 \\
\hline Fetuin-A [ng/mL] & $-0.002 \pm 0.001$ & 0.035 \\
\hline
\end{tabular}

Unstandardized $\beta \pm$ SE values indicate the change in IMT [mm] per 1 - SD change in variables; *Variables included in multivariate models when univariate analysis showed parameters with $p<0.2$ $\mathrm{BP}$ - blood pressure; $\mathrm{FEV}_{1}$ - forced expiratory volume in $1 \mathrm{~s}$; $\mathrm{BP}$ - blood pressure; $\mathrm{FEV}_{1}$ - forced expiratory volume in $1 \mathrm{~s}$;
$\mathrm{FVC}$ - forced vital capacity; HDL — high density lipoprotein cholesterol; LDL - low density lipoprotein cholesterol

first, patients with normotensive COPD had significantly higher cIMT values, higher CRP levels, and lower fetuin-A levels than the control group. Second, there was a negative correlation between cIMT and fetuin-A, and the predictors of increased cIMT were age and fetuin-A levels. Third, ABI values did not differ significantly in patients with COPD compared to the control group and there was also no relationship between $\mathrm{ABI}$ values and fetuin-A levels.

Increased cIMT was shown to be a strong indicator of future CV events [16]. There are many studies on the relationship between incre- 
ased cIMT and COPD; age and $\mathrm{FEV}_{1}$ have also been found to be predictors for increased cIMT in patients with COPD [21-23], as well as other atherosclerotic risk factors such as hypertension, diabetes and dyslipidemia. We did not observe any correlation between cIMT and $\mathrm{FEV}_{1}$. Similar to our results, some previous studies have shown increased cIMT and decreased ABI, an indicator for generalized atherosclerosis, in patients with COPD and in subjects with reduced $\mathrm{FEV}_{1}$ [21-24]. Although it is known that hypertension is one of the most important risk factors for atherosclerosis, cIMT is strongly related not only to systolic BP, but also age and dyslipidemia [25]. In our study, all participants with dyslipidemia were subjected to regular treatment. We assume that the contributing effects of dyslipidemia to cIMT might be running slow in the study participants.

Because $\mathrm{ABI}$ associates with $\mathrm{CAD}$, and defect stenosis $>50 \%$ in major leg arteries [26], we particularly investigated $A B I$ values of all the study subjects to exclude peripheral artery disease. Given that there were no abnormal ABI values in our COPD patients, we assume that this might be related to the condition of their mild and moderate stage of COPD and to non-hypertensive as well as anti-hyperlipidemic treatment. While decreased $\mathrm{ABI}$ values are associated with prominent atherosclerosis [27], increased cIMT values are related potentially to early atherosclerosis [16].

There has been a number of contradictory reports about the relationship between fetuin-A and atherosclerotic process. While most studies showed that fetuin-A was a negative acute-phase reactant and had anti-inflammatory effects [11, 12], some studies claimed that higher fetuin-A levels were associated with ischemic heart disease and insulin resistance [28, 29]. Therefore, more investigation is warranted to determine the role of fetuin-A in the pathophysiology of CV disease. Eraso et al. [13] showed that lower fetuin-A was associated with peripheral arterial disease in type 2 diabetes. It is known that, in fact, hypertriglyceridemia - which might be often a metabolic part of diabetes mellitus - is associated with increased fetuin A levels [30].

Minas et al. [12] suggested that fetuin-A is decreased in COPD, especially during exacerbations. In the study, the patients with COPD had lower fetuin-A levels than the control group. Our results support the idea that the fetuin-A is a negative inflammatory marker for both COPD and atherosclerosis, even though no correlation was found between fetuin-A and $\mathrm{FEV}_{1}$ in our study.
According to our study, fetuin-A level, as well as age, was surprisingly a predictor of increased cIMT. Therefore, in fetuin-A deficiency, the increase in inflammatory conditions and the increase in vascular calcification may play a role as an important contributor to the increased cIMT.

\section{Limitations of the study}

There are several limitations of this study. Firstly, the most important limitation is that we studied a relatively small number of patients with stage 3 and 4 COPD. Secondly, all the patients in the COPD group were receiving regular treatment with inhaled bronchodilators and steroids, and some subjects in both groups were on anti-hyperlipemic agents, which may be a factor in mitigating the impact of fetuin-A on cIMT and ABI, which are markers of atherosclerosis. We did not investigate whether these agents have an effect on the study variables.

\section{Conclusions}

Lower fetuin-A levels are related with COPD, but not with any particular stage of COPD, and are also negatively correlated with cIMT. Additionally, an increased cIMT is associated with COPD and age in non-hypertensive subjects. Further studies are necessary to detect the contribution of the serum inflammatory markers such as IL-6 and TNF, as well as fetuin-A, on the association between COPD and CV risk in a large number of patients with mild, moderate and severe COPD.

\section{Conflict of interest: none declared}

\section{References}

1. Rabe KF, Hurd S, Anzueto A et al. Global Initiative for Chronic Obstructive Lung Disease. Global strategy for the diagnosis, management, and prevention of chronic obstructive pulmonary disease: GOLD executive summary. Am J Respir Crit Care Med, 2007; 176: 532-555.

2. Sin DD, Man SF. Chronic obstructive pulmonary disease: A novel risk factor for cardiovascular disease. Can J Physiol Pharmacol, 2005; 83: 8-13.

3. Boschetto P, Beghé B, Fabbri LM, Ceconi C. Link between chronic obstructive pulmonary disease and coronary artery disease: Implication for clinical practice. Respirology, 2012; 17: 422-431.

4. Koenig W. Inflammation and coronary heart disease: An overview. Cardiol Rev, 2001; 9: 31-35.

5. Spagnoli LG, Bonanno E, Sangiorgi G, Mauriello A. Role of inflammation in atherosclerosis. J Nucl Med, 2007; 48: 1800-1815.

6. Gan WQ, Man SF, Senthilselvan A, Sin DD. Association between chronic obstructive pulmonary disease and systemic inflammation: A systematic review and a meta-analysis. Thorax, 2004; 59: 574-580. 
7. Cesari M, Penninx BW, Newman AB et al. Inflammatory markers and onset of cardiovascular events: Results from the Health $\mathrm{ABC}$ study. Circulation, 2003 11; 108: 2317-2322.

8. van der Meer IM, de Maat MP, Bots ML et al. Inflammatory mediators and cell adhesion molecules as indicators of severity of atherosclerosis: The Rotterdam Study. Arterioscler Thromb Vasc Biol, 2002; 22: 838-842.

9. Schafer C, Heiss A, Schwarz A et al. The serum protein alpha 2-Heremans-Schmid glycoprotein/fetuin-A is a systemically acting inhibitor of ectopic calcification. J Clin Invest, 2003; 112: 357-366.

10. Jahnen-Dechent W, Heiss A, Schäfer C, Ketteler M. Fetuin-A regulation of calcified matrix metabolism. Circ Res, 201; 108: 1494-1509.

11. Wang H, Sama AE. Anti-inflammatory role of fetuin-A in injury and infection. Curr Mol Med, 2012; 12: 625-633.

12. Minas M, Mystridou P, Georgoulias P, Pournaras S, Kostikas K, Gourgoulianis KI. Fetuin-A is Associated with disease severity and exacerbation frequency in patients with COPD. COPD, 2013; 10: 28-34.

13. Eraso LH, Ginwala N, Qasim AN et al. Association of lower plasma fetuin-A levels with peripheral arterial disease in type 2 diabetes. Diabetes Care, 2010; 33: 408-410.

14. Roos M, Oikonomou D, von Eynatten M et al. Associations of fetuin-A levels with vascular disease in type 2 diabetes patients with early diabetic nephropathy. Cardiovasc Diabetol, 2010; 9: 48.

15. Ix JH, Barrett-Connor E, Wassel CL et al. The associations of fetuin-A with subclinical cardiovascular disease in community-dwelling persons: the Rancho Bernardo Study. J Am Coll Cardiol, 2011; 58: 2372-2379.

16. Lorenz MW, Markus HS, Bots ML, Rosvall M, Sitzer M. Prediction of clinical cardiovascular events with carotid intima-media thickness: A systematic review and meta-analysis. Circulation, 2007; 115: 459-467.

17. Smith ER, Ford ML, Tomlinson LA, Rocks BF, Rajkumar C, Holt SG. Poor agreement between commercial ELISAs for plasma fetuin-A: An effect of protein glycosylation? Clin Chim Acta, 2010; 411: 1367-1370.

18. Standardization of Spirometry, 1994 Update. American Thoracic Society. Am J Respir Crit Care Med, 1995; 152: 1107-1136.
19. Orchard TJ, Strandness DE. Assessment of peripheral vascular disease in diabetes. American Heart Association Scientific Statement. Circulation, 1993; 88: 819-828.

20. Weitz JI, Byrne J, Clagett GP. Diagnosis and treatment of chronic arterial insufficiency of the lower extremities: A critical review. Circulation, 1996; 94: 3026-3049.

21. Iwamoto H, Yokoyama A, Kitahara $\mathrm{Y}$ et al. Airflow limitation in smokers is associated with subclinical atherosclerosis. Am J Respir Crit Care Med, 2009; 179: 35-40.

22. Schroeder EB, Welch VL, Evans GW, Heiss G. Impaired lung function and subclinical atherosclerosis. The ARIC Study. Atherosclerosis, 2005;180: 367-373.

23. Beşir FH, Yllmaz Aydın L, Yazgan Ö et al. Evaluation of carotis intima media thickness in chronic obstructive pulmonary disease patients. Tuberk Toraks, 2012; 60: 238-245.

24. Pecci R, De La Fuente Aguado J, Sanjurjo Rivo AB, Sanchez Conde P, Corbacho Abelaira M. Peripheral arterial disease in patients with chronic obstructive pulmonary disease. Int Angiol, 2012; 31: 444-453.

25. Morrison KM, Dyal L, Conner W et al. Cardiovascular risk factors and non-invasive assessment of subclinical atherosclerosis in youth. Atherosclerosis, 2010; 208: 501-505.

26. Hakeem F, Siddique S, Saboor QA. Abnormal ankle brachial index and the presence of significant coronary artery disease. J Coll Physicians Surg Pak, 2010; 20: 79-82.

27. Raman PG, Thakur BS, Mathew V. Ankle brachial index as a predictor of generalized atherosclerosis. J Assoc Physicians India, 2001; 49: 1074-1077.

28. Weikert C, Stefan N, Schulze MB et al. Plasma fetuin-A levels and the risk of myocardial infarction and ischemic stroke. Circulation, 2008; 118: 2555-2562.

29. Lorant DP, Grujicic M, Hoebaus C et al. Fetuin-A levels are increased in patients with type 2 diabetes and peripheral arterial disease. Diabetes Care, 2011; 34: 156-161.

30. Roos M, von Eynatten M, Heemann U, Rothenbacher D, Brenner H, Breitling LP. Serum fetuin-A, cardiovascular risk factors, and six-year follow-up outcome in patients with coronary heart disease. Am J Cardiol, 2010;105: 1666-1672. 